\title{
Aspectos de la construcción del conocimiento sobre la sociedad
}

\section{Aspects of the construction of knowledge about society}

\author{
Juan Delval ${ }^{*}$
}

\begin{abstract}
RESUMEN
El niño tiene que construir modelos o representaciones de la realidad social en la que vive, para tratar de dar un sentido al mundo que le rodea y además esas representaciones sirven de marco para su acción. La mayor parte de esas representaciones mentales no son simples copias de las de los adultos, sino que constituyen una construcción personal y difieren cualitativamente de las de los mayores. El estudio del desarrollo del conocimiento sobre la sociedad en el niño atrae cada vez más atención por parte de los investigadores. Tres son los enfoques teóricos principales. La posición constructivista, según la cual el niño tiene que realizar su propia construcción de los conocimientos con ayuda de los instrumentos intelectuales de que dispone. La tradición del estudio de las representaciones sociales originada en la sociología francesa y renovada por Moscovici, y la psicología histórico-cultural de tradición vygotskiana. La primera posición pone el acento sobre la actividad del sujeto, mientras que las otra dos enfatizan las influencias ambientales y constituyen un necesario contrapunto de la posición constructivista. Sin embargo, la existencia de creencias infantiles diferentes de las de los adultos y del contexto social, y la universalidad de algunas de estas creencias nos obligan a no olvidar la labor personal de construcción que tiene que realizar el sujeto.

Palabras-clave: comprensión infantil de la sociedad; constructivismo; representaciones sociales.
\end{abstract}

${ }^{*}$ Universidad Nacional de Educación a Distancia - Madrid, España. 


\begin{abstract}
The child has to construct models or representations about the reality of the society where he or she lives in order to give a meaning to the surrounding world and, furthermore, taking into consideration that these representations serve as landmarks to his action. Most of the mental representations constructed by the child are not simple copies of the adult representations; instead, they are personal constructions and differ qualitatively from those of the older people. The study of the development of knowledge about society in children is attracting the attention of many investigators. There are three main theoretical approaches to this subject: the constructivist position says that the child has to construct their own knowledge counting on their personal intellectual instruments; the traditional study about social representations originated in the French School of Sociology, that has been renewed by Moscovici; and the Historical Cultural Psychology of vygotskyan approach. The first position emphasizes the activity of the subject, while the others put the emphasis on the environmental influences and so hold a counterpoint to the constructivist position. However, the existence of children beliefs different from those from adults' as well as from the social context, and the universality of some of these beliefs, compel us not to forget the personal work of construction made by the subject.

Key-words: children's understanding of society; constructivism; social representations.
\end{abstract}

\title{
El estudio de las representaciones del mundo
}

Durante mucho tiempo la psicología se ha preocupado sobre todo por estudiar lo que podemos llamar las funciones psicológicas generales, como la percepción, la cognición, las emociones, las imágenes mentales, el lenguaje, la memoria, etcétera. El enfoque de Piaget se centró en estudiar cómo se forman nuevos conocimientos y en la génesis de los mecanismos más básicos que sirven para organizarlos. Su interés era fundamentalmente epistemológico, es decir referente a cómo se produce el conocimiento y por ello estudió las categorías generales que sirven para organizar la realidad.

Pero una de las principales capacidades que poseen los seres humanos es la de construir representaciones de la realidad que les rodea. El conocimiento del ambiente es una forma esencial de adaptación al medio. La posición 
constructivista establece que no podemos conocer la realidad tal y como es sino que construimos modelos de ella, la representamos en nuestra mente, lo que nos permite anticipar lo que va a suceder y actuar de acuerdo con esas representaciones.

La utilización de las categorías de espacio, tiempo y causalidad, es decir, de grandes principios organizadores de la realidad, dota a las representaciones de un alcance inusitado. En efecto, los modelos no sólo permiten explicar lo que sucede, dar sentido a los acontecimientos, sino que hacen posible anticipar lo que va a suceder, y ahí radica su importancia. El espacio permite situar lo que sucede en un lugar, la causalidad establecer relaciones de dependencia entre acontecimientos, pero es el tiempo el que posibilita moverse en una dimensión más abstracta, que dota de significado a la causalidad. No es posible desplazarse realmente en el tiempo, pero si puede hacerse mentalmente y predecir lo que va a suceder, para, de esa forma, ajustar la acción actual a lo que se desea obtener. Disponer de esa prodigiosa capacidad de recordar lo que ha sucedido, y por tanto poder extraer enseñanzas hacia el presente y el futuro de lo que ya pasó, y sobre todo de anticipar lo que todavía no ha tenido lugar constituye el gran éxito del hombre y también su punto más débil. La conciencia de la finitud, el miedo a la muerte, la bondad o maldad de las acciones, depende de esa capacidad para representarse lo que va a suceder.

El estudio de las categorías que nos permiten organizar el conocimiento ha constituido un paso muy importante para entender la mente humana, pero es un estudio que queda muy alejado de lo que la gente piensa y hace en la vida cotidiana. Lo que podemos inferir de la observación más elemental es que cuando un sujeto tiene que resolver un problema o dar una explicación lo hace a partir de la construcción de un modelo de ella. ¿Cómo repartir una tarta redonda entre seis personas? ¿Cómo calentar leche evitando que hierva y se nos derrame? ¿Cómo conseguir que todos los niños vayan a la escuela? ¿Cómo hacer que no haya pobres? ¿Cómo reducir el calentamiento global de nuestro planeta?

Del mismo modo podemos observar que nos movemos perfectamente por nuestra casa, incluso a oscuras, porque tenemos una representación de cómo es, vamos al cine y sabemos lo que tenemos que hacer porque tenemos una representación del cine. Por eso resulta muy importante conocer cuáles son esas representaciones y como se forman.

Podemos decir pues que hay tres enfoques de la construcción del conocimiento:

1) un primer enfoque lo constituye el estudio de las grandes categorías que permiten organizar el conocimiento, Los grandes mecanismos necesarios para conocer. Es lo que se ha denominado la macrogénesis. 
2) un segundo tipo de estudio lo constituyen los caminos que sigue el sujeto en la resolución de tareas concretas cuando aplica esas grandes estructuras en una situación determinada. Es lo que Inhelder y sus colaboradaras han denominado la microgénesis.

3) pero cabe estudiar también las representaciones que el sujeto elabora de grandes o pequeñas parcelas de la realidad.

Podría señalarse que queda un cuarto tipo de estudio que sería el de la conducta que efectivamente realizan los individuos. Pero este tipo de estudio no puede hacerse independientemente y necesita de los anteriores. La conducta directamente observable es demasiado ambigua y puede estar determinada por diferentes estados mentales. Cuando un individuo realiza acciones tan simples como sentarse, ponerse a caminar o golpear a otra persona, no podemos saber por qué lo hace ni qué significado tiene si no tratamos de penetrar en su mente, en sus representaciones de la situación en la que se encuentra.

La construcción de representaciones precisas de la realidad, incluyendo en ella a uno mismo y a los otros, es, sin duda, el mayor logro de la especie humana, su arma más poderosa para controlar la naturaleza. El hombre reconstruye en su mente la realidad, descubre las relaciones entre las cosas, los hechos, traza modelos del funcionamiento de las fuerzas de la naturaleza, de las relaciones físicas entre los objetos, del papel de los otros y de él mismo. Los hombres no se limitan a actuar para satisfacer sus necesidades biológicas, que son irrenunciables e inaplazables, sino que con su mente elabora esos modelos en los que está representado el mundo, lo cual da un sentido más amplio a su actividad y la dota de una eficacia mucho mayor que si se limitara a la pura acción.

Gracias a disponer de sus complejas representaciones o modelos de la realidad el hombre no necesita actuar continuamente para conocer el resultado de sus acciones. A partir de la representación puede anticipar los resultados de su acción, sin necesidad de experimentar lo que va a suceder: puede predecirlo a partir de las características de su modelo del fenómeno o la situación de la que se esté ocupando. Esto le da un enorme poder sobre las cosas y sobre los otros seres vivos. Actuar mentalmente en el marco de una representación es mucho más rápido y más flexible que actuar sobre las cosas. Disponiendo de un buen modelo se puede experimentar de forma más eficaz que si fuera preciso hacerlo de forma material, pues mentalmente pueden manejarse muchas más posibilidades y de forma más completa. Después de haber experimentado mentalmente es cuando puede realizarse el contraste con la realidad, que en todo caso es la última piedra de toque de las predicciones mentales. El lenguaje y la capacidad de utilización de sistemas abstractos de representación aumentan enormemente las posibilidades de actuar mentalmente, pero no es la causa, sino sólo el vehículo en el que expresar esas representaciones. 
El hombre construye representaciones de toda la realidad que le rodea, del funcionamiento de las fuerzas de la naturaleza, de los otros seres vivos y de él mismo. Entre esas representaciones están las de la propia vida social, que incluyen cómo nos relacionamos con los demás, cuál debe ser nuestro comportamiento hacia ellos y qué es lo que esperamos que los demás hagan en las distintas situaciones sociales. El hombre tiene que elaborar entonces modelos del funcionamiento social, de las instituciones en cuyo marco se desarrolla la vida social. Es una labor que tiene que hacer cada individuo, con la ayuda de los otros, basándose en el conocimiento acumulado por las generaciones que le han antecedido, pero que no puede recibir ya hecho. Es, por tanto una labor psicológica, que se realiza en un ámbito social.

A lo largo de su desarrollo los individuos llegan a tener ideas bastante precisas sobre cómo funciona el mundo social, sobre las relaciones con los otros y sobre como están organizadas las instituciones sociales dentro de las que se desenvuelven. Para actuar en la sociedad las personas necesitan adquirir ideas acerca de cómo se produce el proceso de compra-venta y cómo está organizada la sociedad desde el punto de vista económico, para qué sirve el dinero y cuál es su valor; y también entienden la organización política, las relaciones de poder, las formas de gobierno, el funcionamiento de la administración. Pero su conocimiento no se limita a esto, entienden las diferencias de clases sociales, de razas, de países, la función de instituciones como la escuela, la familia, la nación, la religión, los conflictos entre grupos, que dan lugar a las guerras. Y además entienden las relaciones con los otros, las normas que regulan esas relaciones, la conducta que se debe seguir y la que se debe evitar. En definitiva, se forman "representaciones" o "modelos" sobre cómo está organizada y cómo funciona la sociedad, pero también de lo que se debe hacer en distintas situaciones.

Cuando los seres humanos nacen no disponen de esas ideas, que si tienen los adultos, por lo que hay que suponer que las van formando o adquiriendo de alguna manera a lo largo de su periodo de desarrollo, y durante el resto de su vida. Lo que tenemos que examinar es cómo se forman, cómo se "construyen", las ideas sobre el mundo social, sobre las instituciones y sobre las normas que las regulan. Ese es un problema que compete estudiar a la psicología, aunque hay que tener datos de las disciplinas que tienen por objeto estudiar esos distintos aspectos del conocimiento social, como la sociología, antropología, economía, ciencia política. Pero estudiar cómo se forman esas ideas no es un mero entretenimiento o una curiosidad, pues las representaciones del mundo social determinan lo que los sujetos hacen y pueden hacer, cómo actúan. Y para entender las concepciones de los adultos es esencial conocer su proceso 
de formación. Estamos convencidos, por tanto, de que estudiar la génesis de los conceptos sociales tiene una enorme utilidad para entender las ideas adultas sobre la sociedad y que es un requisito indispensable para desarrollar una epistemología genética de las ciencias sociales. En consecuencia podemos decir que el interés del estudio de la formación del conocimiento social es múltiple y puede considerarse desde el punto de vista epistemológico, desde el psicológico o desde el educativo. Para la educación resulta esencial, pues lo que se pretende en la escuela es que los sujetos formen representaciones adecuadas del mundo en que viven por lo que el profesor tiene que partir necesariamente de las ideas que tienen los sujetos si quiere realizar su tarea de un modo satisfactorio.

Las representaciones no tienen sólo una función explicativa, sino que tratan de satisfacer otras necesidades del sujeto, necesidades que no son sólo de tipo cognitivo, sino que contienen aspectos ideológicos, motivacionales o afectivos. La función explicativa se produce para poder alcanzar los fines de la acción, para poder actuar, por lo que las representaciones están indisolublemente ligadas a los fines que se plantea el sujeto, aunque también los determinan, es decir los sujetos persiguen fines en función de las representaciones que tienen, al mismo tiempo que establecen las representaciones para alcanzar sus fines. Se establece así una relación circular.

Uno de los problemas del estudio de las representaciones es que no puede llegarse a ellas directamente, sino sólo de una forma indirecta, infiriendo a partir de lo que hacen o de lo que dicen los sujetos. Podemos considerar las representaciones como el conjunto de propiedades que los individuos atribuyen a una parcela de la realidad, lo que incluye las propiedades de los elementos, las relaciones entre ellos, las explicaciones de por qué acontecen, las relaciones causales, y otras muchas cosas. Además hay que tener en cuenta que las representaciones se establecen para actuar.

Probablemente las representaciones no están completamente listas y disponibles, elaboradas con todas sus partes, en cualquier momento. El sujeto, en el momento en que lo precisa, combina distintos elementos de los que dispone anteriormente de acuerdo con las necesidades del momento, con los fines que persigue. Parecería pues que los elementos están ya ahí, pero el ensamblaje preciso de ellos sólo se realiza para responder a una necesidad que se produce en un momento determinado. Esa necesidad puede ser muy diferentes tipos, puede ser una necesidad de tipo material, para resolver un problema práctico (cómo conseguir ser admitido en un doctorado), o puede ser para explicar un fenómeno que acontece (por ejemplo, por qué quiere separarse mi mujer de mí, o porque está bajando la cotización de las empresas de nuevas 
tecnologías en la bolsa). Para entender esos fenómenos necesito recurrir a las representaciones que tengo, y para ello tengo que elaborar con los elementos de los que ya dispongo una representación adecuada al problema.

Pero lo que me parece cierto es que esas representaciones existen porque las acciones y las explicaciones de los sujetos tienen un carácter bastante unitario, y guardan puntos en común cuando un mismo sujeto trata de explicar diferentes parcelas de la realidad. Esto me lleva a pensar que el conocimiento no está codificado como elementos aislados, sino como bloques más amplios, y que, por tanto, las representaciones existen. Lo que sucede es que una acción no tiene necesariamente que provocar la puesta en marcha de la representación, más que en el caso de que existan elementos discordantes. Cuando voy a encender la luz de mi cuarto no necesito poner en funcionamiento una representación explícita si la luz se enciende al apretar el interruptor, más bien me limito a aplicar un esquema que está automatizado, pero si la luz no se enciende entonces es cuando necesito activar una representación.

\section{Características de las representaciones}

\begin{tabular}{ll} 
Origen & $\begin{array}{l}\text { Los sujetos precisan de representaciones para } \\
\text { sobrevivir en el mundo }\end{array}$ \\
\hline Funciones & $\begin{array}{l}\text { Las representaciones permiten actuar y } \\
\text { entender }\end{array}$ \\
\hline Elaboración & $\begin{array}{l}\text { Se producen como respuesta a la satisfacción } \\
\text { de las necesidades. Por tanto tiene su finalidad } \\
\text { en la acción y la supervivencia. }\end{array}$ \\
\hline Constituyen el & $\begin{array}{l}\text { Las representaciones son lo que está en la } \\
\text { mente de los individuos, son el dato de que } \\
\text { contenido de la } \\
\text { mente }\end{array}$ \\
\hline No son explícitas & $\begin{array}{l}\text { La representaciones no existen de una forma } \\
\text { fijada, más que en casos excepcionales, pues } \\
\text { se va generando a medida que el sujeto las } \\
\text { necesita }\end{array}$ \\
\hline Características & $\begin{array}{l}\text { Pero la representaciones no son específicas } \\
\text { de cada problema, sino que tienen unas } \\
\text { comunes }\end{array}$ \\
características comunes y generales entre \\
ellas, que se muestra sobre todo en el tipo de \\
actuación que realizan los sujetos.
\end{tabular}


Características de las representaciones

\begin{tabular}{ll} 
Evolución & $\begin{array}{l}\text { La formación de representaciones sigue una } \\
\text { serie de estadios regulares }\end{array}$ \\
\hline Importancia & $\begin{array}{l}\text { La representaciones tienen una enorme } \\
\text { importancia desde el punto de vista educativo } \\
\text { pues es lo que los profesores tienen que } \\
\text { contribuir a formar }\end{array}$
\end{tabular}

En todo caso lo que estamos planteando es que uno de los enfoques de la psicología es estudiar las representaciones, su creación, su desarrollo, y sus tipos.

\section{El mundo social}

Si todavía nos queda mucho por conocer acerca de como son las representaciones de los fenómenos físicos, matemáticos, biológicos o químicos, sabemos todavía menos sobre el mundo social. En ese terreno las cosas son mucho más complicadas. Mientras todos pensamos que nuestra representación de la realidad natural es más o menos adecuada, y que nos aproximamos lentamente hacia una verdad que está ahí, independiente de nosotros, en cambio, en el caso del conocimiento de la sociedad es más fácil darse cuenta de que ese conocimiento está orientado por nuestros prejuicios, por nuestros intereses, por nuestros sesgos particulares, por nuestro propio punto de vista, en una palabra, por nuestra posición en el mundo social, como ya había mostrado Marx. El ser rico o ser pobre, poderoso o insignificante, pertenecer a un país o a otro, profesar una religión concreta o no practicar ninguna, ser hombre o mujer, joven o viejo, etc., determina de una manera muy profunda cómo vemos la sociedad y los fenómenos que se producen en ella. Ni tan siquiera las ciencias sociales están al margen de esos sesgos y ésta es una de las causas de la existencia de puntos de vista a veces contrapuestos entre los propios científicos sociales.

Esto añade una dificultad suplementaria a la comprensión del conocimiento sobre la sociedad, respecto al de los fenómenos físicos, pero al mismo tiempo hace especialmente interesante estudiar su génesis. No se trata sólo de un problema de conocimientos escolares, sino que lo importante es 
ver cómo un individuo se hace adulto dentro de una sociedad determinada y va adquiriendo la ideología de esa sociedad; y también cómo va entendiendo las instituciones sociales, las reglas de funcionamiento social, etc.

Cuando preguntamos a los niños sobre problemas de tipo social, aunque sea incidentalmente en conversaciones informales, nos damos cuenta de la dificultad que tienen para entender cosas que a los adultos nos resultan obvias. Nuevamente, como en el caso del conocimiento acerca de la naturaleza, las respuestas de los niños no son simples curiosidades sino, como nos enseñó Piaget, la expresión profunda de su pensamiento. Pero estudiar estas cuestiones es más difícil que estudiar las nociones de la ciencia natural, entre otras cosas porque no tenemos una guía tan firme y segura que nos sirva de punto de referencia. Los conceptos de las ciencias sociales no sólo son muy complejos y a menudo están mal definidos, sino que ni siquiera existe un acuerdo sobre muchos de ellos. No todo el mundo coincide en qué es una nación, qué es la plusvalía, qué debemos considerar terrorismo, qué es un sistema democrático o una dictadura, qué es una clase social, por poner sólo unos pocos ejemplos. En todo caso, no son conceptos de precisión comparable a los de masa, cantidad de movimiento o conjunto, sobre los que caben pocas divergencias.

Esta dificultad explica que hasta hace menos de treinta años se habían realizado pocas investigaciones sobre este tema (ver una breve revisión histórica en Delval, 1989 y una revisión por temas en Furnham; StaceY, 1991 y una visión de conjunto de distintos problemas en BARRETT; BUCHANAN-BARROW, 2005), aunque naturalmente hay trabajos pioneros entre los que destacan los estudios de Piaget sobre el juicio moral (1932) y sobre algunas nociones sociales en relación con problemas lógicos (PiageT, 1924).

Hacia finales de los años 60 y principios de los 70 empezó a surgir un nuevo interés por investigar estos problemas al descubrirse la importancia que tenía para el niño conocer los procesos sociales. La razón del escaso interés que se había manifestado durante años, sobre todo dentro de la psicología anglosajona, puede explicarse por el influjo de la posición conductista, que prestaba poca atención a la organización del conocimiento y además tendía a creer que la conducta del individuo era sobre todo un reflejo del ambiente en el que se desarrollaba. Por ello las investigaciones hasta los años 70 se centraron sobre todo en el origen de las conductas sociales, en el moldeamiento de la conducta social, dentro de la línea de trabajos como los de Sears o Bandura, pero no en el conocimiento social.

El desarrollo de la psicología cognitiva puso de manifiesto la insuficiencia de los análisis que se limitaban exclusivamente a la conducta, así como la necesidad de estudiar el conocimiento como un aspecto indisociable y 
determinante de aquella. Algunos psicólogos, influidos por la psicología de Piaget, señalaron que para el desarrollo social del niño era esencial que formara representaciones de los otros y de si mismo, así como de distintos tipos de relaciones sociales: amistad, autoridad, dependencia, etc. Cuando nos dirigimos a otro tenemos expectativas sobre lo que va a hacer, realizamos anticipaciones de su conducta, y la nuestra está guiada por esas anticipaciones. Construimos modelos de las situaciones y nos comportamos adecuadamente dentro de ellas. No es lo mismo que estemos en la puerta del cine y tratemos de entrar, que llamemos a la puerta de la casa de un amigo, o que veamos que se acerca un guardia tras haber cometido una infracción de tráfico. Aprendemos a reconocer cada una de esas situaciones y a comportamos dentro de ellas de acuerdo con los usos sociales del medio en que vivimos.

Estudiando este tipo de problemas surgió de modo muy floreciente el área que se ha denominado "conocimiento social" ("social cognition" en inglés) y que estudia un ámbito de problemas muy amplio. Brooks-Gunn y Lewis (1978) han dicho que se ocupa del conocimiento de los otros, de uno mismo, y de las relaciones entre uno mismo y los otros. Esto incluiría entonces el conocimiento de los otros en tanto que individuos, con sus deseos, sentimientos, tendencias, etc., así como el conocimiento de nosotros mismos, también como individuos, con nuestros deseos, estados de ánimo, etc.; el conocimiento de las relaciones sociales entre individuos, las relaciones sociales diádicas; las relaciones y el funcionamiento de los grupos sociales más extensos; y finalmente el funcionamiento de las instituciones sociales, tales como las instituciones políticas, la familia, la escuela, las empresas económicas, etc. (SHANTZ, 1982).

Los estudios que se han realizado se pueden agrupar bajo tres grandes rótulos, como hace Turiel (1979):

A) El conocimiento de los otros y de uno mismo. El sujeto va elaborando un conocimiento de las otras personas y de las relaciones con ellas, y a través de él se va conociendo a si mismo. Desde el punto de vista del sujeto se trata más bien de un conocimiento de tipo psicológico, ya que se tienen en cuenta los estados mentales de los otros y se anticipa cómo se van a comportar entre ellos o en relación con el sujeto. Desde el punto de vista de un observador externo puede verse como social porque implica la relación con otras personas, pero desde el punto de vista del sujeto es un conocimiento psicológico. Este tipo de estudios es lo que ha sido abordado por los autores anglosajones bajo la denominación de social cognition. Se estudian relaciones interpersonales como la amistad o la autoridad. Los estudios sobre la llamada "teoría de la mente" pueden encuadrarse en este apartado. Los trabajos sobre la "adopción de perspectivas" (role taking) pueden considerarse también como un componente de ese conocimiento. 
B) El conocimiento moral y convencional. El sujeto va adquiriendo las reglas o normas que regulan las relaciones con los otros. Las normas morales regulan los aspectos más generales de las relaciones interpersonales, mientras que las normas convencionales se ocupan de regulaciones más particulares propias de cada sociedad, como las formas de saludo y cortesía, las costumbres, etc. Estos estudios se originan en los trabajos de Piaget sobre el juicio moral, desarrollados por Kohlberg y otros. La línea de trabajos sobre la conducta y el razonamiento prosocial pueden incluirse también aquí (EISENBERG, 1989).

C) El conocimiento de las instituciones. Los estudios que hemos situado en A) se ocupan de los otros en tanto que personas individuales, pero no como seres sociales que se ha hallan inmersos en instituciones sociales y que se comportan desempeñando "papeles". Lo característico de lo propiamente social, que constituye el objeto de estudio propio de la sociología, lo constituye el conocimiento de las instituciones, es decir de relaciones entre individuos o grupos que transcienden al individuo (Berger; LuCKMANn, 1966). Las relaciones con el tendero, el jefe o el representante político no son relaciones personales, como sería el caso de la amistad, sino que son relaciones entre papeles sociales. Lo que más propiamente podemos llamar conocimiento social es ese conocimiento del funcionamiento de la sociedad en sus distintos aspectos, que es un conocimiento de relaciones institucionalizadas (Delval, 1991).

\section{La formación del conocimiento social}

Estos tres tipos de estudios tienen relaciones entre sí pero también una cierta independencia y siguen tradiciones de investigación distintas. El área del conocimiento de los otros (A) trata de estudiar más que un conocimiento propiamente social un conocimiento de las personas en tanto que sujetos que tienen intenciones, deseos, creencias, etc. Por tanto se trata de un conocimiento sobre todo de naturaleza psicológica, aunque se le haya llamado "social cognition", denominación que no parece muy adecuada.

El estudio del conocimiento propiamente social (C) es el que se refiere a las instituciones, que es quizá el aspecto menos estudiado, y es de este del que nos vamos a ocupar aquí. El conocimiento moral (B), se encuentra entre los dos y constituye un aspecto importante de las relaciones con los otros como individuos y del funcionamiento de las instituciones. 
Las instituciones sociales tienen una característica particular y es que en ellas los individuos desempeñan funciones (tendero, jefe, comprador, asalariado, alumno, ciudadano, etc.) y no actúan como un simple sujeto psicológico. Además las instituciones sociales, que son producto de reglas constitutivas (SEARLE, 1995), lo que hacen es dotar de nuevo significado a fenómenos que ya existen, y además son fruto del acuerdo social (el dinero vale porque todos admitimos que vale) (cf. Delval, 2000)

Cuando se habla de estos temas es frecuente que se produzca una confusión entre el origen del conocimiento y su contenido. Tenemos que manifestar de forma inequívoca que, en nuestra opinión, todo conocimiento tiene un origen social, que el conocimiento sólo es posible viviendo en sociedad y que compartimos con los otros el conocimiento, tanto el que tiene como objeto el mundo inanimado, como el que se ocupa de los seres vivos en general o de los seres humanos en particular. Por tanto tiene que quedar claro que todo conocimiento es social en su origen. Pero el conocimiento puede versar sobre la realidad inanimada, sobre los seres vivos o sobre los humanos y sus relaciones. Es decir que aunque sea social en su origen los contenidos sobre los que trata se pueden referir a cualquier parcela de la realidad.

Pero el hecho de que el conocimiento sea social, de que otros lo posean e intenten transmitírnoslo, de que sea compartido, no quiere decir que se adquiera por copia o transmisión verbal de lo que los otros saben. El sujeto que adquiere un conocimiento no se limita a adquirir lo que otro sabe, sino que lo tiene que reconstruir. De otro modo no se podría explicar que las concepciones de la sociedad de sujetos de distintas edades difieran mucho entre ellas y difieran de las de los adultos, y en cambio se parezcan entre sujetos de parecida edad que viven en diferentes países o culturas.

Sin embargo, algunas explicaciones acerca de como se forma el conocimiento sobre la sociedad sostienen que se trata de un proceso de socialización, que el conocimiento está en la sociedad y es implantado en el individuo por la presión social. Eso es cierto, pero desde el punto de vista del psicólogo lo que hay que explicar precisamente es cómo se produce esa interiorización del conocimiento.

Por ejemplo la teoría de las representaciones sociales propuesta por Moscovici (1976) ha tratado de ocuparse de un tipo de representaciones que serían compartidas por un grupo social de características determinadas. Esas representaciones sociales se adquirirían en la vida social y vendrían hechas del exterior. Como dice Moscovici (1976, p. 66) el individuo forma parte de un "coro" colectivo, que constituye la opinión pública, en el que el individuo participa, lo quiera o no. Por tanto el sujeto es un elemento pasivo que se limita a cantar en ese coro la partitura que recibe socialmente. 
De forma parecida, la psicología histórico-cultural, inspirada en las ideas de Vygotsky, pone también el acento en la adquisición por el individuo de conocimientos que están en la sociedad. Pero de este modo no podemos explicar la originalidad que tienen las concepciones sobre la sociedad que defienden los niños (Delval, 2001).

La concepción de la formación de las representaciones que defendemos es mucho más amplia que la de Moscovici o la de la psicología histórico-cultural $\mathrm{y}$, como veremos, el individuo tiene un papel activo en su elaboración que va mucho más allá de formar parte de un coro o de interiorizar lo que piensan los adultos. Precisamente lo que interesa estudiar son las dificultades que el sujeto encuentra para llegar a las nociones adultas y las etapas por las que pasa.

\section{Los campos de la representación del mundo social}

Después de estas observaciones sobre cómo se va formando el conocimiento social, vamos a tratar de describir qué temas, aspectos o campos pueden incluirse bajo ese rótulo. La representación del mundo social es algo enormemente amplio y con límites difusos, en donde se pueden distinguir aspectos centrales y aspectos periféricos. Posiblemente los dos problemas centrales de la representación del mundo social son la comprensión del orden político y la comprensión del orden económico, que constituyen una especie de columna vertebral en torno a la que se organizan otras cuestiones. Pero además de los problemas que podemos considerar genuinamente sociales hay muchos otros que no lo son estrictamente, pero que tienen una vertiente social clara como la vejez o la muerte. Vamos a enumerar distintos aspectos que forman parte de esa representación del mundo social, que no la agotan, pero que dan una idea de la amplitud de la problemática.

Respecto al funcionamiento económico de la sociedad es importante averiguar cómo se entiende la producción y el intercambio de las mercancías, y ligado a ello, cuál es el papel del dinero. Esto a su vez está en conexión con la distribución social de la riqueza y la existencia de clases sociales y aquí aparecen entonces los problemas de la comprensión de las diferencias sociales, de la estratificación y la movilidad social.

Respecto a la comprensión del orden político, temas centrales son el papel de los partidos políticos, el funcionamiento del sistema democrático y de otros sistemas políticos, de las instituciones, la representación parlamentaria, 
el cambio político, la alternancia en el poder, etc. Un aspecto más profundo del problema, es el de la comprensión de las nociones de autoridad y poder y su extensión desde el punto de vista social. Otro aspecto de gran importancia es la comprensión de las leyes, su origen, su función, su evolución, el papel del derecho en la sociedad y las relaciones entre derecho y moral.

Además de estos dos temas centrales, hay otros muchos aspectos relativos a instituciones o simplemente a fenómenos ligados a la práctica social. Entre estos hay que destacar las ideas referentes a la propia nación, la aparición de un apego hacia el propio país y la comprensión del país como una unidad multidimensional, política, económica, cultural, lingüística, religiosa, geográfica, etc. Muy estrechamente relacionado con esto están las ideas y actitudes hacia otros países, y la aparición de sentimientos negativos como el racismo y la xenofobia.

La concepción de la familia y de su papel dentro de la sociedad, las relaciones de parentesco, las funciones paterna y materna y, en relación con ello, el problema de la adopción de papeles sexuales o de género, constituyen otro de los aspectos importantes de la comprensión de la sociedad. La adopción de papeles sexuales ha sido estudiada tradicionalmente desde la perspectiva de la socialización mientras que se han descuidado los aspectos cognitivos del problema, es decir, cómo perciben los sujetos su papel y el de los individuos que pertenecen a otro sexo.

Ligada a la existencia de clases sociales, que tiene un fundamento económico, aparece la cuestión de la adopción de papeles sociales, la comprensión de las profesiones y todo lo relativo a la división del trabajo, lo cual se conecta con los progresos del niño en la diferenciación entre los individuos y su papel social.

Los niños pasan una gran cantidad del tiempo en la escuela y construyen ideas sobre cuál es la función de ésta y qué es lo que hacen en ella. El problema de la transmisión del saber, de la generación de conocimientos desde el punto de vista social y de la difusión de esos conocimientos, así como el papel de la ciencia como forma de poder, es otro de los aspectos de nuestro tema, pero que ha sido muy escasamente estudiado.

El nacimiento y la muerte además de fenómenos biológicos son fenómenos sociales, los jóvenes, los adultos y los viejos tienen un papel en la sociedad, y cada sociedad les otorga un lugar. La visión de las etapas de la vida, desde la infancia hasta la vejez, en relación con la organización social, constituye otra parte del campo que nos ocupa. 
La guerra y la paz, así como las relaciones entre las naciones en todos los terrenos, forman un aspecto más de este campo que debe ser estudiado. Los niños se interesan enormemente por la guerra como forma de dominación, pero tienen de ella un conocimiento irreal y esquemático. El paso de esa concepción a las ideas complejas de los adolescentes, que incluyen aspectos políticos, económicos y sociales en la conceptualización de la guerra, es un tema que vale la pena estudiar.

La religión constituye también un fenómeno social y las ideas que el niño tiene en el terreno religioso no coinciden con las de los adultos. Es importante por ello saber cuáles son esas ideas de los niños, cómo conciben a Dios, o aspectos más mundanos, como son su pertenencia a una comunidad religiosa, en una palabra, cómo es la religión del niño.

Por último, un problema central, que aparece ligado a todos los anteriores, es la comprensión del cambio social, cómo evolucionan las sociedades a lo largo de la historia. Este tema está en relación con el del tiempo histórico, algo que resulta incomprensible para el niño durante largos años. Los niños tienden a ver la sociedad de forma estática y el tiempo sólo aparece tardíamente como un elemento relacionado con los fenómenos sociales. Una compresión completa de la sociedad exige entender la evolución histórica de las sociedades.

Los distintos campos de la representación del mundo social tienen características diferentes y grados de dificultad desigual. En algunos casos se trata simplemente de comprender regulaciones sencillas sobre ciertos usos sociales. En otros hay que entender sistemas simples, muchos de cuyos aspectos pueden comprenderse de una forma concreta. Esto sucede, por ejemplo, con muchas vertientes de la idea de país. En cambio, en otros casos se trata de entender un sistema muy amplio que está formado a su vez por subsistemas que interactúan, como en el caso de la organización económica o la organización política de la sociedad, que resulta muy compleja de entender y su comprensión parece exigir instrumentos de tipo formal.

Cada uno de los distintos campos requiere el empleo de los instrumentos intelectuales que el niño forma a través de su acción sobre el mundo y uno de los problemas de interés en este estudio es ver cómo interactúan los medios intelectuales del sujeto con los conceptos sociales que forma y si esa interacción es del mismo tipo que la que se produce respecto a los conceptos físicos o matemáticos (véase una revisión más extensa en Delval, 1989 o Furnham; StACEY, 1991; sobre nociones económicas BeRTI; BOMBI, 1981/88; ver también Furth, 1980; Delval; Enesco; Navarro, 1994). 


\section{De que están hechos los modelos sociales}

Las representaciones sobre el mundo social tienen algunas características peculiares que las diferencian de otras representaciones, debido al carácter propio del conocimiento social. Es especialmente importante el papel que las normas y los valores desempeñan en la vida social. Las normas sociales regulan la vida social pero, a diferencia de las reglas de la naturaleza, pueden respetarse o no respetarse (Delval; ENEsco, 1994).

Puesto que el hombre es un ser social que sólo puede desarrollarse dentro de una sociedad y en contacto estrecho con los otros, los adultos tratan de que los niños se conviertan en miembros completos de esa sociedad inculcándoles las normas, valores, actitudes y formas de comportamiento que caracterizan a los miembros de esa sociedad, en un proceso que globalmente se suele llamar socialización, y que no presupone necesariamente cómo se produce, si mediante la presión de los otros o a través de la actividad constructiva del propio sujeto.

Una de las primeras cosas que los sujetos adquieren son las normas o reglas sobre lo que debe hacerse y sobre lo que no debe hacerse. Los adultos se cuidan mucho de que la conducta de los niños siga esas normas y ponen un gran empeño en ello, pues esto es lo que va a garantizar que en el futuro su conducta pueda considerarse social, y que el sujeto pueda interaccionar con los otros. Por ello desde muy pronto se les anima o se les reprime para que se comporten de acuerdo con lo que se considera adecuado. Esas normas están estrechamente ligadas a valores sociales que indican lo que es deseable y lo que no lo es desde el punto de vista de los otros. Esos elementos prescriben lo que debe hacerse y se refieren a cómo deben ser las acciones, no a cómo son. Normas y valores son constituyentes esenciales del conocimiento y de la conducta social.

Pero el niño recibe además informaciones sobre muchos hechos sociales, sobre aspectos concretos de la realidad social, y también las obtiene él mismo actuando dentro del mundo social, registrando sus regularidades y reflexionando sobre él.

Junto con todo lo anterior, y apoyándose en ello, va elaborando explicaciones sobre cómo y por qué suceden las cosas de una determinada manera, en definitiva, sobre el funcionamiento de los sistemas sociales.

Normas, valores, informaciones y explicaciones son entonces algunos de los elementos que componen los modelos o representaciones que el niño 
va elaborando sobre el mundo social, elementos que podemos agrupar en tres tipos: normativo-valorativos, informativos y explicativos. Quizá esta distinción pueda parecer trivial pero no tenerla presente da lugar a muchos equívocos en las investigaciones y sobre todo en lo referente a cómo se produce el conocimiento social. Intentaremos explicar por qué.

Las normas y los valores que prescriben lo que se debe hacer se empiezan a adquirir desde muy pronto y los adultos ponen un particular empeño en que los niños los adquieran. ("No pegues a los otros niños", "para comprar en la tienda hay que llevar dinero", "tienes que ir a la escuela para aprender", serían ejemplos de ello). Por lo tanto se transmiten explícitamente y se estimula su imitación, de tal manera que su labor mental en los comienzos de la adquisición de las normas es relativamente pasiva ya que los sujetos las reciben en gran medida hechas. En este caso la transmisión directa por parte de los adultos desempeña un papel importante y podría pensarse que el niño se limita a incorporar lo que los adultos le transmiten, pero, de todas formas, el niño sólo es capaz de incorporar aquellas normas que están al alcance de su comprensión.

En muchos casos las informaciones las recibe también directamente de los adultos o de los medios de comunicación y además la escuela dedica una importante parte de su actividad a la transmisión de informaciones, aunque también el niño las busca por sí mismo. (Ejemplos de estas serían: "Brasilia es la capital de Brasil", "Benito Juárez fue presidente de México", "médico es una profesión prestigiosa", "la bandera colombiana es blanca, azul y roja".)

Por el contrario las explicaciones sobre por qué son así las cosas, sobre cómo funcionan los sistemas sociales, y los conceptos en que se apoyan, apenas se enseñan. No se le explica al niño el sistema de la tienda y la distribución de mercancías, ni sus relaciones con el proceso de producción. Ni tampoco se le explica cómo se produce la movilidad social y cuáles son sus determinantes, ni las causas que motivan las guerras. Y cuando se le enseñan esas cosas hace ya mucho tiempo que tiene explicaciones para ellas, explicaciones que ha tenido que construir por si mismo, porque nadie se las ha dado, y que suponen un trabajo de elaboración propio. El niño las construye con los instrumentos intelectuales de que dispone y llega a explicaciones que no coinciden con las de los adultos y que curiosamente son muy semejantes entre niños de distintos medios sociales y de diferentes países, aunque pudiera creerse lo contrario.

Así vemos que el niño aprende muy pronto como una regla que para comprar hay que llevar dinero a la tienda, mucho antes de que sea capaz de explicar para qué sirve ese dinero, qué hace el tendero con él, cómo se fijan los precios, y en general cómo tiene lugar la actividad económica. Posteriormente 
las explicaciones inciden sobre normas y valores y los reorganizan, y cuando se alcanza un grado de comprensión grande, esas explicaciones proporcionan a las normas y valores un sentido nuevo, haciendo posible la reflexión sobre ellos, e incluso dudar de sus fundamentos.

Algo semejante puede decirse de las informaciones. A nuestros niños se les enseña al llegar a la escuela que "Madrid es la capital de España" $\mathrm{y}$ todos saben repetirlo sin errores. Pero, como han puesto de manifiesto varias investigaciones (Piaget; Weil, 1951), y nosotros mismos hemos estudiado, antes de los siete años no suelen entender que hay más españoles que madrileños, que no todos los españoles son madrileños, pero si sucede lo contrario, y tampoco tienen la más remota idea de lo que quiere decir "ser capital de" ni lo que es una capital, ni un país (Delval; Del Barrio; Echeíta, 1981; Delval, 1989). Así pues, esa información no les sirve de mucho en ese momento, ni pueden integrarla en sus explicaciones de la organización administrativa de un país.

Consideramos que es útil tener presentes las distinciones anteriores porque cuando estudiamos los modelos del mundo social que elaboran los sujetos, si nos interesamos especialmente por su conocimiento de las reglas, valores e informaciones no podemos apreciar plenamente la labor constructiva del sujeto, que en gran medida está reproduciendo lo que se le ha transmitido. Por ello lo que nos dice depende mucho del medio social y de la cultura en la que vive, de las ideas dominantes, aunque también las está asimilando con sus instrumentos intelectuales. En cambio, en las explicaciones sobre el funcionamiento de los sistemas sociales y en los conceptos que forman parte de ellas, las variaciones son menores y encontramos procesos constructivos que son mucho más universales. Es fácilmente comprensible que los contenidos del pensamiento tienen que variar de acuerdo con el entorno, pero la manera de explicar los fenómenos, que está mucho más ligada a las capacidades mentales de los sujetos, es más semejante.

\section{REFERÊNCIAS}

BARRETT, M.; BUCHANAN-BARROW, E. (Eds.). Children's understanding of society. Hove: Psychology Press, 2005. 
BERGER, P. L.; LUCKMANN, T. La construcción social de la realidad. Buenos Aires: Amorrortu, 1968.

BERTI, A. E.; BOMBI, A. S. Il mondo economico nel bambino. Firenze: La Nuova Italia, 1981/ 88. Trad. inglesa, revisada y ampliada: The child's construction of economics. Cambridge: Cambridge University Press, 1988.

BROOKS-GUNN, J.; LEWIS, M. Early social knowledge: the development of knowledge about others. In: MCGURK, H. (Ed.). Issues in childhood social development. Londres: Methuen, 1978.

DELVAL, J. La representación infantil del mundo social. In: ENESCO, I.; TURIEL, E.; LINAZA, J. (Eds.). El mundo social en la mente infantil. Madrid: Alianza, 1989.

. Notas sobre la construcción del conocimiento social. In: HINOJAL, J. A. et al. (Comps.). Sociedad, cultura y educación: homenaje a la memoria de Carlos Lerena Alesón. Madrid: CIDE y Universidad Complutense, 1991. p. 191-208.

. Sobre la naturaleza de los fenómenos sociales. In: KORTA, K.; GARCÍA MURGA, F. (Comps.). Palabras. Víctor Sánchez de Zavala in memoriam. Bilbao: Servicio Editorial de la UPV-EHU, 2000. p. 95-122.

. Vygotsky, Piaget: a formação do conhecimento e a cultura. Educação $e$ Realidade, Porto Alegre, v. 26, n. 2, p. 89-126, jul./dic. 2001.

DELVAL, J.; DEL BARRIO, C.; ECHEITA, G. El conocimiento de los niños de su propio país. Cuadernos de Pedagogía, n. 75, p. 33-36, 1981.

DELVAL, J.; ENESCO, I. Moral, desarrollo y educación. Madrid: Anaya-Alauda, 1994.

DELVAL, J.; ENESCO, I.; NAVARRO, A. La construcción del conocimiento económico. In: RODRIGO, M. J. (Comp.). Contexto y desarrollo social. Madrid: Síntesis, 1994. p. 345-383.

EISENBERG, N. The development of prosocial values. In: EISENBERG, N.; REYKOWSKI, J.; STAUB, E. (Eds.). Social and moral values: individual and societal perspectives. Hillsdale: Erlbaum, 1989.

FURNHAM, A.; STACEY, B. Young people's understanding of society. Londres: Routledge, 1991.

FURTH, H. G. The world of grown-ups. Children's conceptions of society. Nueva York: Elsevier North Holland, 1980. 
MOSCOVICI, S. La psychanalyse, son image et son public. París: P.U.F., 1976.

PIAGET, J. Le jugement et le raisonnement chez l'enfant. Neuchâtel: Delachaux y Niestlé, 1924.

. (1932) Le jugement moral chez l'enfant. 4. ed. París: Alcan, P.U.F., 1969.

PIAGET, J.; WEIL, A. M. Le development, chez l'enfant, de l'idée de patrie et des relations avec l'étranger. Bulletin International des Sciences Sociales, n. 3, p. 605-621, 1951.

SEARLE, J. R. The construction of social reality. Nueva York: Free Press, 1995.

SHANTZ, C. U. Children's understanding of social rules and the social context. In: SERAFICA, F. C. (Ed.). Social cognitive development in context. Nueva York: The Guilford Press, 1982.

TURIEL, E. (1979) Distinct conceptual and developmental domains: social convention and morality. Nebraska Symposium on Motivation. Lincoln: University of Nebraska Press, 1977. v. 25.

Texto recebido em 28 jan. 2006 Texto aprovado em 15 abr. 2007 